\title{
Impact of intercostal nerve blocks using liposomal versus standard bupivacaine on length of stay in minimally invasive thoracic surgery patients
}

\author{
Dana A. Dominguez ${ }^{1}$, Sora Ely ${ }^{1}$, Cynthia Bach ${ }^{2}$, Tina Lee ${ }^{2}$, Jeffrey B. Velotta ${ }^{2}$ \\ ${ }^{1}$ Department of Surgery, University of California, San Francisco, CA, USA; ${ }^{2}$ Kaiser Permanente Medical Center, Oakland, CA, USA \\ Contributions: (I) Conception and design: C Bach, JB Velotta, T Lee; (II) Administrative support: JB Velotta, T Lee; (III) Provision of study materials \\ or patients: All authors; (IV) Collection and assembly of data: DA Dominguez, S Ely, C Bach; (V) Data analysis and interpretation: All authors; \\ (VI) Manuscript writing: All authors; (VII) Final approval of manuscript: All authors. \\ Correspondence to: Jeffrey B. Velotta, MD. Department of Surgery, 3600 Broadway, Oakland, CA 94602, USA. Email: Jeffrey.B.Velotta@kp.org.
}

\begin{abstract}
Background: Postsurgical pain control can have a significant impact on patient outcomes and hospitalassociated costs. We sought to evaluate the effect of intercostal nerve blocks using liposomal bupivacaine on length of stay (LOS) in patients undergoing video-assisted thoracoscopic surgery (VATS).

Methods: We retrospectively reviewed outcomes in 80 patients undergoing VATS wedge resection, VATS lobectomy, or minimally-invasive esophagectomy (MIE). Patients received either liposomal bupivacaine $(n=40)$ or standard-release bupivacaine with epinephrine $(n=40)$ via intercostal nerve block. The LOS, 24-hour postoperative pain scores, overall opioid usage, and patient ambulation rates at 24 hours were compared for the two groups.

Results: The median LOS was significantly shorter in patients receiving liposomal bupivacaine, at 1.35 days (IQR, 1.28 to 1.53 days) compared to 2.45 days (IQR, 2.08 to 3.51 days) in patients receiving standard-release bupivacaine $(\mathrm{P}<0.0001)$. Average post-operative pain score during the first 24 hours was $3.4 \pm 1.8$ for the liposomal bupivacaine group and $2.3 \pm 1.2$ for the control group $(\mathrm{P}=0.002)$. This difference, though statistically significant, is likely not clinically significant, as there was no difference in 24-hour postoperative intravenous morphine equivalent usage between the liposomal bupivacaine and control groups (29.8 \pm 21.0 vs. $31.9 \pm 20.9 \mathrm{mg}$, respectively, $\mathrm{P}=0.664)$. Interestingly, however, $93 \%(37 / 40)$ of patients receiving liposomal bupivacaine were able to ambulate within 24-hours after surgery, compared to $65 \%(26 / 40)$ of patients in the control group $(\mathrm{P}=0.003)$.

Conclusions: The use of liposomal bupivacaine is associated with decreased LOS in postoperative thoracic surgery patients and earlier return to ambulation. It does not, however, decrease 24-hour postoperative pain scores or opioid usage.
\end{abstract}

Keywords: Liposomal bupivacaine; regional anesthesia; thoracoscopy; length of stay (LOS)

Submitted Aug 15, 2018. Accepted for publication Oct 17, 2018.

doi: $10.21037 /$ jtd.2018.10.124

View this article at: http://dx.doi.org/10.21037/jtd.2018.10.124

\section{Introduction}

Pain control after surgery is vital in preventing postoperative complications. Patients with adequate pain control are better able to mobilize, take deep breaths, cough, and clear secretions. These maneuvers are thought to prevent complications such as deep vein thrombosis, atelectasis, and pneumonia. In addition, management of acute pain after surgery is critical in preventing development of chronic postsurgical pain (1). Historically, thoracic epidural analgesia has been the gold standard for pain control after thoracotomy, but it is commonly associated with undesirable side effects including hypotension and urinary retention. 
For minimally-invasive video-assisted thoracoscopic surgery (VATS), intercostal nerve blocks can be used as an alternative to epidurals for pain control (1). Although initial pain control is adequate with this approach, it often requires supplementation with additional therapies due to the short half-life of local anesthetics.

Liposomal bupivacaine $\left(\operatorname{Exparel}^{\circledR}\right)$ is a long-acting analgesic that is FDA-approved for local wound infiltration into the surgical site (2). Liposomal bupivacaine is composed of a unique DepoFoam ${ }^{\circledR}$ carrier matrix that encapsulates bupivacaine and slowly releases medication over a period of time (3). Liposomal bupivacaine has a duration of action of up to 96 hours, compared to bupivacaine with epinephrine, which lasts only $8-12$ hours $(4,5)$.

Early studies on regional application of liposomal bupivacaine described possible benefits of decreased length of stay (LOS) and postoperative pain in patients who had undergone breast reconstruction, hemorrhoidectomy, or hip arthroplasty $(6,7)$. More recently, in the thoracic surgery literature, a study by Khalil et al. concluded that in patients undergoing open thoracotomy, the use of liposomal bupivacaine in intercostal nerve blockade provided significantly better pain control in postoperative days 1 and 3 , compared to epidural analgesia (8). Additionally, Rice et al. found that posterior intercostal nerve blockade with liposomal bupivacaine provided effective analgesia and was a suitable alternative to thoracic epidural analgesia for VATS patients in a study that included VATS, robotic-assisted thoracic surgery (RATS), or thoracotomy approaches (9). Both studies found a decreased LOS for patients receiving liposomal bupivacaine compared with patients receiving thoracic epidurals $(8,9)$.

The purpose of this retrospective study was to evaluate the impact of liposomal bupivacaine on LOS in patients receiving VATS surgery. Although previous studies compared liposomal bupivacaine to thoracic epidurals, our study assessed the difference in LOS between the use of liposomal bupivacaine and standard-release bupivacaine for intercostal nerve blockade.

\section{Methods}

\section{Study design}

This study was a retrospective cohort analysis completed at the Kaiser Permanente Oakland Medical Center. The study was approved by the Kaiser Permanente Northern
California Institutional Review Board (IRB: CN-152502-H).

The primary outcome of this study was LOS in thoracic surgery patients undergoing VATS wedge resection, VATS lobectomy, or minimally-invasive esophagectomy (MIE), compared between patients who received liposomal bupivacaine and patients who received bupivacaine with epinephrine $(1: 100,000)$. Intercostal nerve block was performed at the end of each operation prior to chest tube placement under direct VATS visualization. For the liposomal bupivacaine group, $266 \mathrm{mg}(20 \mathrm{~mL}$ drug diluted with $0.9 \%$ normal saline to total volume of $30 \mathrm{~mL}$ ) was injected in equal amounts in successive rib spaces from one above and one below the outermost port site rib spaces. For the bupivacaine with epinephrine group, an equivalent dose of $0.25 \%$ bupivacaine with epinephrine $(1: 100,000)$ was used with the identical technique. Secondary outcomes included 24-hour postoperative pain scores, opioid utilization, and time to ambulation. Additionally, 30-day emergency department (ED) visits and hospital readmissions were recorded.

\section{Selection of study patients}

Patients who received VATS were identified using Epic HealthConnect, the Kaiser Permanente electronic medical record. Eligible patients were at least 18 years of age and underwent one of three common VATS procedures (wedge resection, lobectomy, or MIE) performed by the same three Kaiser Permanente Oakland Medical Center board-certified thoracic surgeons from October 2013 to October 2015. Included patients received either liposomal bupivacaine or bupivacaine with epinephrine as an intercostal nerve block. The bupivacaine with epinephrine group are included as historical controls, as the study period included a transition to liposomal bupivacaine as a consistent practice at our institution. This transition represented a complete adoption of liposomal bupivacaine, therefore there was no temporal overlap in patient groups, minimizing possibility for selection bias. Patients were excluded if they underwent a different type of surgery, had a different operating surgeon, or required an admission to the intensive care unit (ICU) after surgery (as the majority of our thoracic surgery patients go to the floor postoperatively, ICU admission was thought to indicate more serious issues that would preclude relevant study of the outcome variables). 
Table 1 Baseline characteristics of patients who underwent VATS

\begin{tabular}{lccc}
\hline Characteristics & LBV $(\mathrm{n}=40)$ & $\mathrm{BPV}(\mathrm{n}=40)$ & $\mathrm{P}$ \\
\hline Age (years), mean $\pm \mathrm{SD}$ & $67 \pm 9.8$ & $69 \pm 11.3$ & 0.383 \\
Male, $\mathrm{n}(\%)$ & $17(42.5)$ & $19(47.5)$ & 0.653 \\
BMI, mean \pm SD & $28.4 \pm 5.8$ & $25.6 \pm 4.9$ & $0.019^{*}$ \\
Comorbidities, $\mathrm{n}(\%)$ & & & \\
Diabetes & $10(25.0)$ & $4(10.0)$ & 0.078 \\
COPD & $4(10.0)$ & $2(5.0)$ & 0.675 \\
CKD & $5(12.5)$ & $9(22.5)$ & 0.239 \\
Cardiovascular disease & $8(20.0)$ & $9(22.5)$ & 0.785 \\
HTN & $21(52.5)$ & $22(55.0)$ & 0.823 \\
Overweight/obese & $27(67.5)$ & $24(60.0)$ & 0.485 \\
\hline
\end{tabular}

*, $\mathrm{P}<0.05$. LBV, liposomal bupivacaine; BPV, standard release bupivacaine with epinephrine; $\mathrm{BMI}$, body mass index; COPD, chronic obstructive pulmonary disease; CKD, chronic kidney disease; HTN, hypertension; VATS, video-assisted thoracoscopic surgery.

Table 2 Characteristics of video-assisted thoracoscopic surgical procedures

\begin{tabular}{lccc}
\hline Characteristics & LBV $(n=40)$ & BPV $(n=40)$ & $P$ \\
\hline $\begin{array}{l}\text { Length of surgery (hours), } \\
\text { mean } \pm \text { SD }\end{array}$ & $3.02 \pm 1: 21$ & $3.20 \pm 1.17$ & 0.296 \\
Type of surgery & & & \\
Esophagectomy & 5 & 7 & 0.529 \\
$\quad$ Number & $4.23 \pm 0.45$ & $5.22 \pm 0.57$ & 0.072 \\
Length of surgery & & & \\
Wedge resection & 14 & 14 & 1.000 \\
$\quad$ Number & $1.54 \pm 0.32$ & $2.25 \pm 0.41$ & 0.056 \\
$\quad$ Length of surgery & & & \\
Lobectomy & 21 & 19 & 0.653 \\
$\quad$ Number & $3.38 \pm 1.19$ & $3.21 \pm 0.46$ & 0.657 \\
\hline
\end{tabular}

LBV, liposomal bupivacaine; BPV, standard release bupivacaine with epinephrine.

\section{Outcome measurement}

All data points collected for analysis were extracted from HealthConnect. LOS was determined by subtracting the date and time of hospital discharge from the date and time of hospital admission. The electronic document flowsheet was used to collect 24-hour postsurgical pain scores and ambulation data for each patient. Time to ambulation was defined by time from case end to time of bedside nurse documentation of first ambulation, which is routinely documented in the medical record. The electronic medication administration record was used to account for any opioid medications administered within 24 hours after surgery, and the use of opioids was quantified as intravenous morphine equivalents $(\mathrm{mg})$. All ED visit or hospital readmissions within 30 days after postsurgical discharge were examined.

\section{Statistical analysis}

A power analysis was performed based on an estimated mean LOS of $2.75 \pm 1.58$ days for standard release bupivacaine and $2.1 \pm 1.25$ days for liposomal bupivacaine group. To detect an absolute difference of 1 day in LOS at $80 \%$ power and alpha of 0.05 we would need a total of 64 patients. Data are reported as means and standard deviations, medians and interquartile ranges (IQR), or percentages. Non-parametric Wilcoxon Rank-sum tests or t-tests were used for analysis of continuous variables; chi-squared tests or Fisher Exact tests were used for categorical variables. Data management and analyses were performed using Statistical Analysis Systems (SAS) 9.4 (Cary, NC). A P value of $<0.05$ was considered statistically significant.

\section{Results}

\section{Baseline patient demographics and characteristics}

The average age was $67 \pm 9.8$ years old for patients in the liposomal bupivacaine group and $69 \pm 11.3$ years old for patients in the standard-release bupivacaine group $(\mathrm{P}=0.383)$. Patients in the liposomal bupivacaine group had a significantly higher average body mass index compared to patients in the control group $\left(28.4 \pm 5.8\right.$ vs. $25.6 \pm 4.9 \mathrm{~kg} / \mathrm{m}^{2}$, $\mathrm{P}=0.019)$ but no difference in the prevalence of diabetes (10 vs. 4, $\mathrm{P}=0.078)$. A complete summary of baseline characteristics is detailed in Table 1 .

The average length of surgery, for all surgeries combined, was $3.02 \pm 1.21$ hours for patients in the liposomal bupivacaine group and $3.20 \pm 1.17$ hours for patients in the who received standard-release bupivacaine $(\mathrm{P}=0.296)$. The numbers of each type of VATS surgery were similar between the two groups. There was no significant difference in length of surgery between the two 


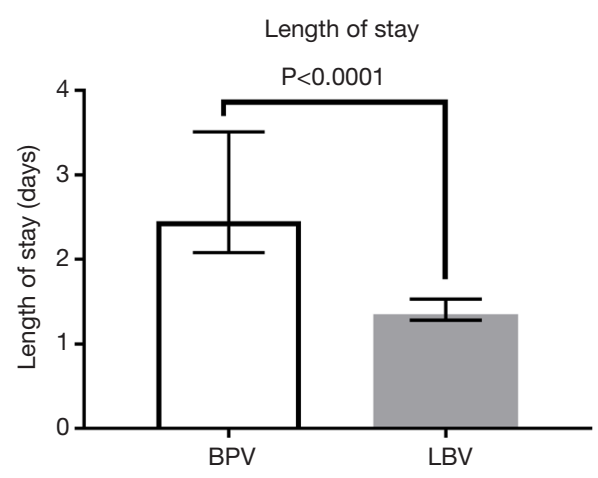

Figure 1 LOS (in days) after thoracic surgery for patients who received liposomal bupivacaine compared to those who received standard release bupivacaine with epinephrine. LOS, length of stay; LBV, liposomal bupivacaine; BPV, standard release bupivacaine with epinephrine.

Table 3 LOS, ambulation and readmission visits

\begin{tabular}{lccc}
\hline Outcome & LBV, n (\%) & BPV, n (\%) & P \\
\hline LOS, days & & & $<0.0001^{*}$ \\
$\geq 2$ & $6(15.0)$ & $32(80.0)$ & \\
$<2$ & $34(85.0)$ & $8(20.0)$ & \\
Ambulation at 24 hours & & & $0.003^{*}$ \\
Yes & $37(92.5)$ & $26(65.0)$ & \\
No & $3(8.5)$ & $14(35.0)$ & \\
Readmissions or ED visits & & & \\
Readmit/ED & $10(25.0)$ & $8(20.0)$ & \\
No & $30(75.0)$ & $32(80.0)$ & \\
\hline
\end{tabular}

*, P<0.05. LOS, length of stay; LBV, liposomal bupivacaine; BPV, standard release bupivacaine with epinephrine; ED, Emergency Department.

groups. A complete summary of surgical characteristics is detailed in Table 2.

\section{Primary outcome measurement}

The median LOS was decreased for patients receiving liposomal bupivacaine by more than a day, at 1.35 days (IQR, 1.28 to 1.53 days) compared to 2.45 days (IQR, 2.08 to 3.51 days) for patients receiving standard-release bupivacaine (Figure 1), and this difference was statistically significant $(\mathrm{P}<0.0001)$. Compared to six patients in the liposomal bupivacaine group, 32 patients in the standard-release bupivacaine group had a LOS of 2 days or longer (Table 3).

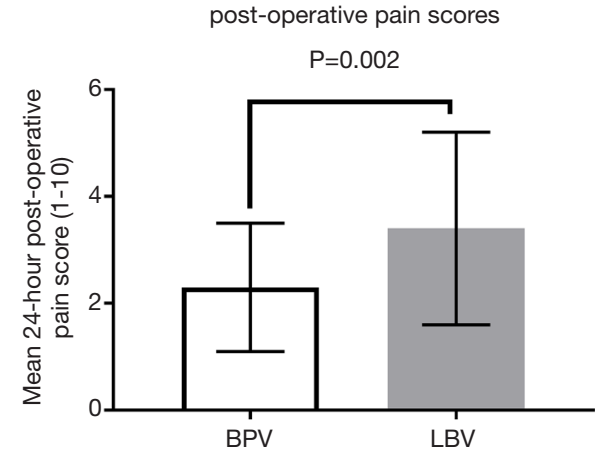

Figure 2 Postoperative pain scores (0 to 10 ) during the first 24 hours after thoracic surgery. LBV, liposomal bupivacaine; BPV, standard release bupivacaine with epinephrine.

$\%$ Ambulating at 24 hours postop

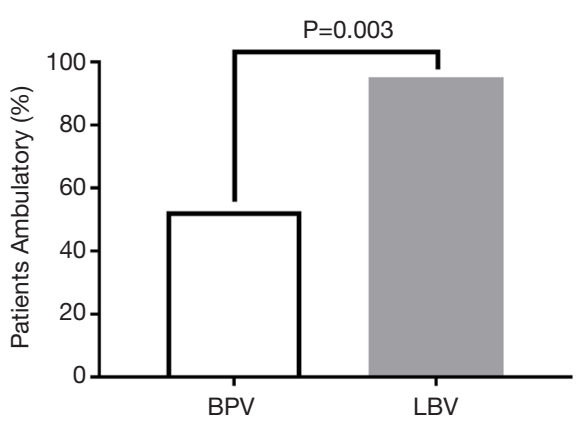

Figure 3 Percent of patients ambulating at 24 hours. LBV, liposomal bupivacaine; BPV, standard release bupivacaine with epinephrine.

\section{Secondary outcome measurements}

Average 24-hour postoperative pain score was $3.4 \pm 1.8$ for the liposomal bupivacaine group and $2.3 \pm 1.2$ for the standard-release group $(\mathrm{P}=0.002)$ (Figure 2). Despite this statistically significant finding, the experimental group did not use more opioid medication and returned to ambulation sooner. There was no significant difference in 24-hour postoperative intravenous morphine equivalent usage between the two groups $(29.8 \pm 21.0$ vs. $31.9 \pm 20.9 \mathrm{mg}$, $\mathrm{P}=0.664)$. However, at 24 -hours postoperatively, $93 \%$ (37/40) of patients receiving liposomal bupivacaine were ambulatory, compared to only $65 \%(26 / 40)$ of patients in the control group $(\mathrm{P}=0.003)$ (Figure 3). Statistical significance was maintained for each group with regards to LOS in the subgroup analysis. Only lobectomy patients had a significant difference in the proportion 
Table 4 Subgroup analysis by surgical procedure

\begin{tabular}{|c|c|c|c|}
\hline Type of surgery & $\operatorname{LBV}(n=40)$ & BPV $(n=40)$ & $\mathrm{P}$ \\
\hline LOS, median (IQR) & $1.57(1.45-1.63)$ & $4.37(4.11-5.27)$ & $0.019^{*}$ \\
\hline Ambulation, n (\%) & $4(80.0)$ & $5(71.4)$ & 1.000 \\
\hline 24-hour morphine eq, mean (SD) & $31.16(15.88)$ & $45.71(22.02)$ & 0.213 \\
\hline LOS, median (IQR) & $1.34(1.30-1.43)$ & $2.07(1.38-2.28)$ & $0.031^{*}$ \\
\hline Ambulation, n (\%) & $13(92.8)$ & $11(78.6)$ & 0.596 \\
\hline 24-hour morphine eq, mean (SD) & $31.27(21.81)$ & $27.15(20.94)$ & 0.615 \\
\hline Lobectomy, n & 21 & 19 & \\
\hline 24-hour morphine eq, mean (SD) & $28.53(22.25)$ & 30.23 (19.23) & 0.798 \\
\hline
\end{tabular}

*, P<0.05. LOS, length of stay; LBV, liposomal bupivacaine; BPV, standard release bupivacaine with epinephrine; SD, standard deviation; $\mathrm{Q}$, quartile range.

of patients ambulating at 24-hours in the liposomal bupivacaine group $(95.2 \%$ vs. $52.6 \%, \mathrm{P}=0.003)$. There was no significant difference between the number of ED visits or hospital readmission rates between the two groups $(\mathrm{P}=0.592)$ (Table 3).

\section{Discussion}

Currently, there is a paucity of data in the literature regarding the impact of liposomal bupivacaine on thoracic surgery patient care. To our knowledge, this study represents the first data describing use of liposomal bupivacaine on patients undergoing VATS lobectomy and esophagectomy. The results of our study suggest that the use of liposomal bupivacaine for intercostal nerve block is associated with a decreased LOS in patients undergoing VATS wedge resection, VATS lobectomy, or MIE. Decreasing LOS has the potential to provide multiple benefits to patients including decreased risk of hospital acquired infections and increased patient satisfaction. Other studies have shown that early hospital discharge for surgery patients results in significant hospital cost savings without apparent increase in morbidity or mortality (10). By decreasing LOS, liposomal bupivacaine has the potential for significant cost savings. We hypothesized that liposomal bupivacaine would decrease LOS by primarily through enhanced postsurgical pain control.
Interestingly, in our study, the average pain scores were slightly higher for patients who received liposomal bupivacaine compared to controls, and the difference was statistically significant. However, the clinical significance of this difference is unclear. Overall pain scores were low for both groups and the small absolute difference in pain scores did not translate to a difference in total intravenous morphine equivalents between the two groups. One possible explanation of this equivalent opioid use is that there are temporal differences in opioid use that are not detected by our study. It is possible that those in the standard release group would use less opioids initially when the drug is active, requiring more as the effect dissipates. One would then expect the opposite in the liposomal bupivacaine group as the drug reaches peak effect. Our results contrast with a recent study that found decreased morphine equivalents at 24 hours in patients undergoing VATS wedge resections, but no concomitant decrease in hospital stay (11). Those findings are not wholly in contradiction to our study, in which the contribution of decreased LOS from the wedge resection patients was minimal because the majority were discharged on postoperative day one. As the selected study populations clearly have distinct differences in the complexity of surgical procedure and recovery, we performed a subgroup analysis to ensure that each group maintained statistical significance for LOS as shown in Table 4. Our findings 
were more consistent with another study, which found that the LOS was decreased by one day in patients who received liposomal bupivacaine compared to those who received an epidural, but did not find a significant difference in average pain scores and opioid consumption (9). A third study found that the average LOS for post-thoracotomy patients was 1.9 days less for those receiving liposomal bupivacaine than for those receiving epidural analgesia; as in our study, there was no difference in supplemental opioid use, but the mean 24-hour pain score was significantly lower for patients who received liposomal bupivacaine (8).

Significantly more of our patients who received liposomal bupivacaine could ambulate within 24-hours after surgery compared to those who received standardrelease bupivacaine. This factor may be contributing to the shorter LOS, as early ambulation after surgery has been shown to result in improved patient outcomes and decreased postsurgical complications (12). In contrast, it may also contribute to the increased subjective pain scores found in our analysis. Given that the patients receiving liposomal bupivacaine ambulated earlier, it is possible that they experienced increased discomfort due to earlier mobilization. Early postoperative mobilization not only prevents complications such as deep venous thromboembolism and/or pulmonary embolism, but for thoracic surgery patients specifically, improves pulmonary function and promotes lung expansion $(13,14)$. Interestingly, on subgroup analysis (Table 4), this effect on return to ambulation appeared to be primarily from the lobectomy group which maintained statistical significance. We believe this is due to the differences in surgical complexity between groups, specifically the fact that wedge resections are often discharged prior to 24 hours and esophagectomy patients have additional abdominal wounds that may contribute to decreased ambulation.

Although patients who received liposomal bupivacaine were discharged from the hospital earlier than patients who received standard bupivacaine, there was no significant difference between the rate of 30-day hospital readmissions and ED visits. Among the patients in the liposomal bupivacaine group, none of the ED visits or readmissions were due to uncontrolled pain, whereas there was one readmission for pain in the standard bupivacaine group. There were no specific trends or common reasons for 30-day hospital readmission or ED visits between the two groups.

\section{Limitations}

This single-center, retrospective study was limited to the data available and documented in the electronic medical record and certainly the use of historical controls carries a risk of temporal and selection bias. The approximate duration of action of liposomal bupivacaine is 96 hours; however, because patients had an average LOS of 1.352.45 days, it was not possible to collect and compare pain scores and opioid use beyond 24 hours after surgery. Due to the extended duration of liposomal bupivacaine, there could potentially be a decrease in pain scores and opioid use beyond 24 hours. Pain scores are highly subjective and although they were documented during each nursing shift, the specific number of pain scores documented per patient varied.

\section{Conclusions}

The use of liposomal bupivacaine as an intercostal nerve block in minimally invasive thoracic surgery may contribute to a decreased LOS. Although this study did not show improved pain scores or decreased opioid utilization, it did find that significantly more patients receiving liposomal bupivacaine were able to ambulate within 24 hours after surgery. Early ambulation may contribute to decreased postsurgical complications and shorter LOS, but the direct impact of these factors with liposomal bupivacaine needs to be confirmed in a larger, prospective, randomized control trial.

\section{Acknowledgements}

Special thanks to Mary Anne Armstrong and Julia Wei in the Kaiser Permanente Division of Research for statistical analysis, as well Pamela Derish in the UCSF Department of Surgery for editorial assistance.

Funding: Research funding provided by Kaiser Permanente Division of Research Graduate Medical Education Grant.

\section{Footnote}

Conflicts of Interest: The authors have no conflicts of interest to declare.

Ethical Statement: The study was approved by the Kaiser Permanente Northern California Institutional Review Board (IRB: CN-15-2502-H). Written informed consent was waived by the Kaiser Permanented IRB as this was a retrospective chart review and posed minimal risk to the patients. 


\section{References}

1. Gottschalk A, Cohen SP, Yang S, et al. Preventing and treating pain after thoracic surgery. Anesthesiology 2006;104:594-600.

2. Pacira Pharmaceuticals I. Exparel Product Insert. San Diego, CA, 2015. Available online: https://www.exparel. com/sites/default/files/EXPAREL_PI_Nerve_Block_ Final_4-6-2018.pdf

3. Pacira Pharmaceuticals I. Exparel. San Diego, CA. 2016. Available online: http://www.exparel.com/index.shtml

4. Exparel. Lexi-comp Online. Available online: http://www. crlonline.com.ucsf.idm.oclc.org/lco/action/doc/retrieve/ docid/patch_f/3548445

5. Marcaine. Lexi-comp Online. Available online: http:// www.crlonline.com.ucsf.idm.oclc.org/lco/action/doc/ retrieve/docid/patch_f/6481

6. Butz DR, Shenaq DS, Rundell VL, et al. Postoperative Pain and Length of Stay Lowered by Use of Exparel in Immediate, Implant-Based Breast Reconstruction. Plast Reconstr Surg Glob Open 2015;3:e391.

7. Domb BG, Gupta A, Hammarstedt JE, et al. The effect of liposomal bupivacaine injection during total hip arthroplasty: a controlled cohort study. BMC Musculoskelet Disord 2014;15:310.

8. Khalil KG, Boutrous ML, Irani AD, et al. Operative

Cite this article as: Dominguez DA, Ely S, Bach C, Lee $\mathrm{T}$, Velotta JB. Impact of intercostal nerve blocks using liposomal versus standard bupivacaine on length of stay in minimally invasive thoracic surgery patients. J Thorac Dis 2018;10(12):6873-6879. doi: 10.21037/jtd.2018.10.124
Intercostal Nerve Blocks With Long-Acting Bupivacaine Liposome for Pain Control After Thoracotomy. Ann Thorac Surg 2015;100:2013-8.

9. Rice DC, Cata JP, Mena GE, et al. Posterior Intercostal Nerve Block With Liposomal Bupivacaine: An Alternative to Thoracic Epidural Analgesia. Ann Thorac Surg 2015;99:1953-60.

10. Calligaro KD, Dougherty MJ, Raviola CA, et al. Impact of clinical pathways on hospital costs and early outcome after major vascular surgery. J Vasc Surg 1995;22:649-57; discussion 657-60.

11. Parascandola SA, Ibanez J, Keir G, et al. Liposomal bupivacaine versus bupivacaine/epinephrine after videoassisted thoracoscopic wedge resectiondagger. Interact Cardiovasc Thorac Surg 2017;24:925-30.

12. Das-Neves-Pereira JC, Bagan P, Coimbra-Israel AP, et al. Fast-track rehabilitation for lung cancer lobectomy: a fiveyear experience. Eur J Cardiothorac Surg 2009;36:383-91; discussion 391-2.

13. Kalisch BJ, Lee S, Dabney BW. Outcomes of inpatient mobilization: a literature review. J Clin Nurs 2014;23:1486-501.

14. Yeung WW. Post-operative care to promote recovery for thoracic surgical patients: a nursing perspective. J Thorac Dis 2016;8:S71-7. 